\title{
Design and Research of a New secure Authentication Protocol in GSM networks
}

\author{
Ai-qin $\mathrm{QI}^{1, a}$, Yong-jun SHEN ${ }^{2}$ \\ ${ }^{1}$ Northwest University For Nationalities ,China \\ ${ }^{2}$ Lanzhou University , China
}

\begin{abstract}
As the first line of defense in the security application system, Authentication is an important security service. Its typical scheme is challenge/response mechanism and this scheme which is simple-structured and easy to realize has been used worldwide. But these protocols have many following problems In the GSM networks such as the leakage of user indentity privacy, no security protection between home registers and foreign registers and the vicious intruders' information stealing and so on. This paper presents an authentication protocol in GSM networks based on maths operation and modular square root technique. The analysis of the security and performance has also been done. The results show that it is more robust and secure compared to the previous agreements.
\end{abstract}

\section{Introduction}

Global mobile communications network GSM originated in 1980 s as the Pan-European digital cellular system standard and it is now accepted as the worldwide wireless communication. They convey all sorts of data and messages through wireless channel and can't require the cables. With the development of network applications, security problems arises. As the first defense line of secure application system, the authentication is the most important secure service. However, it is not feasible for GSM networks to use traditional security mechanisms because of its inherent limitations. A kind of simple and efficient authentication protocol needs to be taken into account.

In this paper, we propose a suitable GSM authentication protocol that use mathematical and modular square residual to complete the authentication process. The protocol security analysis has been done. Compared to the previous agreements, it is more robust and secure.

The rest of the paper is organized as follows: Section 2 discusses related work. Section 3 presents the proposed authentication protocol. Section 4 analyzes the security and the performance of the proposed protocol. Section 5 finally concludes the paper.

\section{Related work}

User authentication is an important topic for communication security and there are many schemes existed for the purpose in the literatures. In 1981, Lamport ${ }^{[1]}$ proposed a user authentication scheme for communication in insecure channel. With the development and application of wireless networks and mobile technologies, people begin to focus on GSM authentication protocol. In 2004, M. S. Hwang and L. $\mathrm{H}$. $\mathrm{Li}^{[2]}$ presented an authentication scheme, but it was found that this solution had weakness and a modified version to overcome them has been presented by M. S. Hwang, I.E.Liao and C. C. Lee ${ }^{[3]}$. The study in the literature [4] has showed that the scheme in [3] also fails to provide anonymity. Two recent studies ${ }^{[5][6]}$ has shown that all above schemes are incapable of providing the anonymity. Later security weaknesses of the approaches in [2-4] have been concluded and an enhanced authentication scheme ${ }^{[7]}$ with anonymity named as EASA has been presented. Through the analysis of the protocol in the literature [7], we find that the scheme is still not to provode anonymity, it is also vulnerable to insider attack, off-line password guessing attack and forgery attack etc.

In this paper, we propose a suitable GSM authentication protocol using mathematical and modular square residual ${ }^{[8]}$ to complete the certification process. This paper also makes the analysis of security and performance. Compared to the previous agreement, it is more robust and secure.

\section{The proposed protocol}

Supposing that a mobile user $\mathrm{Mu}$ whose home agent is $H A$ will visit a foreign network and the foreign agent is $F A$. $H A$ Selects two large prime numbers $\mathrm{p}, \mathrm{q}$ and calculates

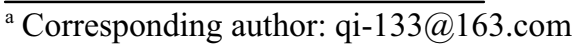


$\mathrm{n}=\mathrm{pq}$ as the public key. $H A$ selecsts a random number $X_{H A}$ and a primitive $\mathrm{g}$ of the multiplicative group $\mathrm{Zn} *$ and calculates $Y_{H A}=g^{X_{H A}} \bmod n .\left(X_{H A}, Y_{H A}\right)$ makes up the master key of $H A$ and the key length is long enough such as 512 bits. For each foreign agent, $H_{A}$ calculates the corresponding session key which meets $S K_{H F}=h\left(I D_{F A}\left\|X_{H A}\right\| I D_{H A}\right)$ by using Diffie-Hellman key distribution protocol and hash function such as SHA-1 algorithm.

In this section, we present our authentication protocol which has five phases: initialization ,login, authentication , refreshment of session key and refreshment of password. Table 1shows the symbol that would be used in this paper.

Table 1. the symbol description

\begin{tabular}{|c|c|}
\hline symbol & Description \\
\hline $\mathrm{Mu}$ & mobile user \\
\hline$I D_{x}$ & The identity of mobile user $\mathrm{x}$ \\
\hline$p w_{u}$ & The passward of mobile user $\mathrm{Mu}$ \\
\hline $\mathrm{HA}$ & Home register of mobile user \\
\hline $\mathrm{FA}$ & Fforeign register \\
\hline$\|$ & Connection operation \\
\hline$\oplus$ & Exclusive-OR operation \\
\hline$h(\cdot)$ & Hash function \\
\hline
\end{tabular}

\subsection{Initialization}

Mobile user Mu sends his identity $I d_{U}$ to his home register HA by a secure channel. HA computes $T H_{u}=h\left(I D_{m u} \| Y_{H A}\right), S K_{u H}=h\left(I D_{m u} \| X_{H A}\right) \quad$ and injects the information $\left\{T H_{u}, S K_{u H}, h(), n.\right\}$ into the smart card. Then HA sends the smart card to the mobile user $\mathrm{Mu}$. After receiving the smart card, $\mathrm{Mu}$ selects a random password $p w_{u}$, a random number $\mathrm{b}$ and calculates $S K_{u H}{ }^{\prime}=h\left(I D_{m u} \| p w_{u}\right) \oplus S K_{u H}, V_{u H}=7 H_{u} \oplus h\left(I_{m u} \| h\left(p w_{u} \| b\right)\right)$, $H_{u H}=h\left(T H_{u}\right) \oplus h\left(I D_{m u} \oplus b\right) . \quad\left\{V_{u H}, H_{u H}, S K_{u H}{ }^{\prime}, h(), n, b.\right\}$ is the final information of the smart card.

\subsection{Login}

$\mathrm{Mu}$ inserts the smart card into the terminal and inputs $I D_{m u}, p w_{u}$ to calculate $T H_{u}^{*}=V_{u H} \oplus h\left(I_{m \imath} \| h\left(p w_{u} \| b\right)\right)$, $H_{u H}{ }^{*}=h\left(T H_{u}^{*}\right) \oplus h\left(I_{m u} \oplus b\right)$. Mu checks if $H_{u H} *$ equals $H_{u H}$, if equal the user is a legal user. Then Mu selects a random number $n_{m u}$,computes

$m_{1}=\left(I_{m u}\left\|D_{F A}\right\| T H_{u} \| n_{m \imath}\right)^{2} \bmod n$ and sends $\mathrm{m}_{1}$ to the foreign agent $F A$.

\subsection{Authentication}

The authentication phase includes five steps as follows:

Step 1: After receiving the information $\mathrm{m}_{1}$, FA selects a random number $n_{F A}$ and computes $r e q_{F A}=n_{F A} \oplus S K_{H F}$ and sends the information $m_{2}=\left\{m_{1}, r e q_{F A}, h\left(m_{1}\left\|r e q_{F A}\right\| S K_{H F}\right)\right\}$ to HA.

Step 2: After receiving FA's message, HA decrypts $\mathrm{m}_{2}$ by using the prime p,q to get $I_{m u}, I D_{F A}, T H_{u}, n_{m u}$. Then HA checkes the validity of $I D_{m u}$ and judges if $h\left(I D_{m u} \| Y_{H A}\right)$ equals $T H_{u}$. If correct, HA computes $h\left(I_{F A}\left\|D_{H A}\right\| X_{H A}\right)$ and checks if $h\left(m_{1}\left\|r e q_{F A}\right\| h\left(I D_{F A}\left\|D_{H A}\right\| X_{H A}\right)\right)$ calculated equals $h\left(m_{1}\left\|r e q_{F A}\right\| S K_{H F}\right)$ received from FA, if the above condition holds, FA is authenticated by HA. HA computes $n_{F A}{ }^{*}=h\left(I D_{F A}\left\|I D_{H A}\right\| X_{H A}\right) \oplus r e q_{F A}$ $K_{1}=h\left(I D_{m u}\left\|I D_{F A}\right\| n_{m u}\right) \oplus h\left(S K_{H F} \| n_{F A}^{*}\right)$ $K_{2}=h\left(h\left(n_{m}\right)\left\|h\left(n_{F A}^{*}\right)\right\| S K_{u H}\right), S_{1}=h\left(K_{1}\left\|K_{2}\right\| n_{F A} * \| S K_{H F}\right)$ and sends $m_{3}=\left\{K_{1}, K_{2}, S_{1}\right\}$ to FA.

Step3: When receiving the message $m_{3}$, FA uses the session key $S K_{H F}$ to compute $h\left(K_{1}\left\|K_{2}\right\| n_{F A} \| S K_{H F}\right)$ and judge if it equals $S_{1}$. If it holds, HA is authenticated by FA. FA calculates $h\left(I D_{m u}\left\|I D_{F A}\right\| n_{m u}\right)=K_{1} \oplus h\left(S K_{H F} \| n_{F A}\right)$, $K_{3}=h\left(I D_{m u}\left\|I D_{F A}\right\| n_{m u}\right) \oplus h\left(n_{F A}\right)$ and sends $m_{4}=\left\{K_{2}, K_{3}\right\}$ to the mobile user $\mathrm{Mu}$.

Step4: $\mathrm{Mu}$ receives the message $\mathrm{m}_{4}$, computes $h\left(n_{F A}\right)=h\left(I D_{m \iota}\left\|I D_{F A}\right\| n_{m u}\right) \oplus K_{3} \quad$ and judeges if $h\left(h\left(n_{m u}\right)\left\|h\left(n_{F A}\right)\right\| S K_{u H}\right)$ equals $K_{2}$, if it holds, FA is authenticated by $\mathrm{Mu}$. Mu computes the session key $S K=h\left(h\left(n_{F A}\right) \| h\left(I D_{m u}\left\|I D_{F A}\right\| n_{m u}\right)\right)$ which is used to communicate with FA. Mu computes $h\left(h\left(n_{F A}\right)-1\right)$ amd sends $m_{5}=h\left(h\left(n_{F A}\right)-1\right)$ to FA.

Step5: FA computes $h\left(h\left(n_{F A}\right)-1\right)$ with the number $n_{F A}$ he selected and compares if $h\left(h\left(n_{F A}\right)-1\right)$ equals $m_{5}$. If equal, $\mathrm{Mu}$ is authenticated by FA. Then FA computes the session key $S K=h\left(h\left(n_{F A}\right) \| h\left(I D_{m u}\left\|I D_{F A}\right\| n_{m u}\right)\right)$ to be used to communicate with $\mathrm{Mu}$. The authentication proceess ends. 


\subsection{Refreshment of Session key}

When $\mathrm{Mu}$ wants to communicate with FA frequently, the session key needs to update periodically. FA sends a temporary certificate $\left(\text { TCert }_{u}\right)_{S K}$ that includes life time and other informations to $\mathrm{Mu}$. If $\mathrm{Mu}$ wants to visit FA in ith time, Mu sends $\left\{\right.$ TCert $\left._{u},\left(n_{i} \| \operatorname{TCert}_{u}\right)_{S K_{i}}\right\}$ to FA. The i-th sessinon key $S K_{i}=h\left(n_{i-1} \| h\left(I D_{m u}\left\|I D_{F A}\right\| n_{m \imath}\right)\right), i=1,2, \ldots, n$ $\left(n_{0}=h\left(n_{F A}\right)\right)$. FA checks the validity of the certificate decrypts $\left(n_{i} \| \operatorname{TCert}_{u}\right)_{S K_{i}}$ with $S K_{i}$ and compares if TCert $_{u}$ equals the certificate decrypted. If it holds, FA saves $n_{i}$ as the next communication. The session key refreshment ends.

\subsection{Refreshment of password}

$\mathrm{Mu}$ inserts the smart card into the terminal to verify the legitimacy of the identity Mu. If it holds, Mu selects a new password $p w^{\prime}$ and a new random number $b^{\prime}$ and computes $\left.\quad V_{u H^{*}}{ }^{*}=T H_{u} \oplus h\left(I D_{m u}\right) \| h\left(p w^{\prime} \| b^{\prime}\right)\right)$, $T H_{u}^{*}=V_{u H}{ }^{*} \oplus h\left(I_{m u} \| h\left(p w^{\prime} \| b^{\prime}\right)\right), \quad H_{u H}{ }^{*}=h\left(T H_{u}^{*}\right) \oplus h\left(I D_{m u} \oplus b^{\prime}\right)$. The smart card information is $\left\{V_{u H}{ }^{*}, H_{u H}{ }^{*}, S K_{u H}{ }^{*}, h(), n,. b^{\prime}\right\}$ and the refreshment of password ends.

\section{Security and performance analysis}

\subsection{Security Analysis}

In Our scheme, the anonymity of $\mathrm{Mu}$ is obtained by hash function and Exclusive-OR operation. Assume that an attacker has extracted the information stored in Mu's smart card and the used meaages tranmitted among Mu,FA and HA. However, the attacker can not derive the real identity of Mu without knowing the value of $(p, q)$ and the random number $n_{m u}$.

In our scheme, the information of the smart card is is $\left\{V_{u H}, H_{u H}, S K_{u H}{ }^{\prime}, h(), n, b.\right\}$. When the user's smart card is stolen or losses , an attacker can guess the user's identity and password to compute $S K_{u H}$ " and $T H_{u}{ }^{*}$. In order to verify the correctness of $S K_{u H}$ " and $T H_{u}{ }^{*}$, the attacker must derive the information from $\left\{m_{1}, m_{2}, m_{3}, m_{4}, m_{5}\right\}$, but the malicious attacker does not know the value of (p, q). According to the residual knowledge of modular square, it is not possible to get the message $m_{1}, n_{m u}, I D_{m u}$, $T H_{u}$ in a polynomial time. So $S K_{u H}$ " and $T H_{u}{ }^{*}$ can not be derived from the information $\left\{m_{1}, m_{2}, m_{3}, m_{4}, m_{5}\right\}$. Our scheme can resist offline password guessing attack

Our scheme also can resist a foreign agent attack. When A malicious foreign agent such as $F A_{j+1}$ replays the used message $m_{1}$ to a legitimate foreign agent such as $F A_{j}$, in this case, $F A_{j+1}$ will get the message $m_{4}=\left\{K_{2}, K_{3}\right\}$, but he does not know the news $h\left(I D_{m u}\left\|I D_{F A}\right\| n_{m u}\right)$ and can't get $h\left(n_{F A}\right)$, so he can not send a response message $m_{5}=h\left(h\left(n_{F A}\right)-1\right)$. Malicious agents can not mimic legitimate agent identity to deceive mobile users because malicious agents do not know the key $S K_{H F}$ and the random number $n_{F A}$.He can not generate messages $m_{4}$ to the user, the attack failed.

Our scheme also can resist forgery attack. Supposing that malicious attackers forgery into a mobile user or home agent to try to attack. They don't know $\left\{T H_{u}, I D_{m u}\right\}$, malicious attackers can not finish the legitimate user authentication through the home agent. They do not know the key (p, q), malicious attackers can not impersonate the home agent to decrypt the message $m_{1}$ and perform the authentication through mobile users and foreign agent.

The proposed protocol also can resist known-session key attack, internal attack and replay attack. This scheme also has the characteristics of forward secrecy and so on, which enhance the performance of the protocol.

\subsection{Performance Analysis}

Our proposed protocol contains only five news exchange in terms of communication load and does not need clock synchronization mechanism, which reduces the consumption of clock synchronization and the timestamp setting. In terms of computational complexity, our protocol uses hash function and Exclusive-OR operation because of the wireless environment restrictions on energy and computing power. The proposed protocol performs not more than 2 msec in OpenSSL running time. Compared with other relevant protocols, our protocol is more simple and efficient.

\section{Conclusions}

In this paper, we propose an efficient authentication protocol for GSM networks and analyze the security and performance of the protocol. The results show that the protocol is secure and robust.

\section{Acknowledgement}

The work is supported by Scientific and technological projects for Central colleges and universities. (No. 31920150079). 


\section{References}

1. 1.L .Leslie, Comm.ACM 24, 770 (1981).

2. J.Zhu, J.Ma, IEEE.Tran.C.E 50,230(2004).

3. C.C.Lee,M.S.Hwang ,I.E.Liao, IEEE. Tran.C.E 53, 1683(2006).

4. C. C. Wu, W.B.Lee, W. J. Tsaur, IEEE. Com.L 12 , 22(2008).

5. J. S.Lee, D. H. Lee, IEEE.Com. L 13, 292(2009).

6. P. Zeng,Z.Cao,K.K.R.Choo, S.Wang, IEEE.Com.L 13,70(2009).

7. C.C.Chang,C.Y.Lee ,Y. C. Chiu,Com.Stan.I 32,611(2009).

8. J.Y.Liu, Applied cryptography(2008). 